\title{
Analysis of Electromagnetic Shielding Stability in electronics shelter
}

\author{
Ting He $\mathrm{H}^{\mathrm{a}}$ Xi Duan ${ }^{\mathrm{b}}$
}

The 28th Research Institute of China Electronics Technology Group Corporation, Nanjing, Jiangsu, 210007, China

atinghelw@163.com, bduanxi1985@163.com

Keywords:Electromagnetic shielding, Shelter Stability, Application

Abstract: First briefly illustrates the importance of electronic shelter shielding stability, and introduces the principle of electromagnetic shielding; through the calculation of the electromagnetic shielding, summarizes the factors affecting the effectiveness of electromagnetic shielding shelter; finally put forward reasonable scheme of shielding effectiveness and stability.

\section{Introduction}

Shelter as a universal loading platform, has good battlefield mobility, rapid response capability, suitable for onboard equipment and personnel work environment, and has the ability to secure their electromagnetic protection of personnel and information, make its application more and more widely in various industries in [1]. With the rapid development of microwave technology, the electromagnetic environment of battlefield in modern war is increasingly complex, the stability of shielding effectiveness is an important index in the environment of modern warfare. Therefore, it is important to study the stability of electromagnetic shielding effectiveness.

\section{Electromagnetic shielding mechanism}

When the electromagnetic wave transferred to a metal plate, it formed the incidence reflection phenomenon at the interface because of electromagnetic wave impedance mutation. The electromagnetic wave energy which was reflected by the shield was named "Reflection attenuation R", the heat loss of electromagnetic wave which through the shield was named "Absorption loss A", the loss of electromagnetic wave which is reflected between the sides of the shield was named "Reflection loss B". Attenuation of electromagnetic energy is the shielding effectiveness.

\section{Shielding Type \& Influencing factors}

According to the electromagnetic wave of frequency, electromagnetic shielding can be divided into magnetic shielding, telectric field shielding, and electromagnetic shielding.. According to the distance between the wave source and the shelter, electromagnetic shielding can be divided into near-field shielding and far-field shielding.

\section{Magnetic shielding}

According to the frequency of the source, the magnetic shielding can be divided into low frequency magnetic shielding and high frequency magnetic shielding. The shielding principle of low-frequency magnetic shielding is diverted the magnetic wave.. ferromagnetic materials can reduce the magnetic leakage..electromagnetic induction phenomenon produced in the shield surface is shielding principle of high frequency magnetic shielding. electromagnetic induction can offset magnetic wave. Thus it can choose strong magnetic material (such as iron, steel and other metal), 
conductor material in low frequency magnetic field ;it can choose low resistivity (such as copper and aluminum)in high frequency magnetic field,. Magnetic shielding in the electromagnetic shielding, is the highest difficulty.

\section{electric field shielding}

The electric field shield is divided into electrostatic shielding and alternating electric field shielding. In electrostatic shielding electrostatic induction can offset equivalent electric charges; the shielding principle of alternating electric fielding can be used to describe the circuit theory, through capacitive coupling between the shelter to reduce the interference source. The essence of electric field shielding is to cut off the power line between the interference source and the sensitive source. The electric field shielding is relatively simple in the whole electromagnetic shielding, and the good conductor is usually chosen.

\section{Electromagnetic shielding}

In electromagnetic shielding, electric field and magnetic field component always exist at the same time. especially increasing of frequency, it can produce electromagnetic radiation. Thuselectromagnetic shielding should reduce the electric wave and magnetic wave. electromagnetic shielding can choose good conductor material, in addition to the high frequency skin effect, conductor thickness is not too thick, as long as the mechanical strength can meet. Moreover, wavelength of interference source is small, and the aperture has a great influence on electromagnetic shielding effectiveness[2].

Classification and influencing factors is shown in Table 1

Table 1 Classification and influencing factors of electromagnetic shielding

\begin{tabular}{cccc}
\hline Shielding Type & Magnetic shielding & Electric field shielding & $\begin{array}{c}\text { Electromagnetic } \\
\text { shielding }\end{array}$ \\
\hline Frequency range & Constant Magnetic & $1 \mathrm{MHz} \sim 500 \mathrm{MHz}$ & $500 \mathrm{MHz} \sim 40 \mathrm{GHz}$ \\
Leaking structure & $\sim 500 \mathrm{KHz}$ & shielding & $/$ \\
Effect factor & shielding & Shielding making thickness \\
& $\begin{array}{c}\text { Interferenceperformance } \\
\text { Interference distance }\end{array}$ & ground resistance & Aperture \\
& & & \\
\hline
\end{tabular}

\section{factors affecting the effectiveness of electromagnetic shielding analysis}

When the electromagnetic wave is transmitted to the metal plate, the shielding effectiveness of the metal plate is:

$$
S E=A+R+B
$$

The absorption loss A is calculated as:

$$
A=1.31 t\left(f u_{r}\right)^{1 / 2}
$$


The reflection loss $R$ is not only related to the characteristics of the material itself, but also with the location of the field source and the metal plate, the following formula:

$$
R=20 \lg \left[\frac{1.173}{r \sqrt{\frac{f \sigma_{r}}{\mu_{r}}}}+0.54 r \sqrt{\frac{f \sigma_{r}}{\mu_{r}}}+0.354\right]
$$

When $A>15 d B$, multiple reflection loss factor can be ignored, otherwise the formula is

$$
B=20 l \mathrm{~g}\left(1-e^{-2 t \bar{\delta}}\right)
$$

In the formula , $t$ is the shielding thickness $(\mathrm{cm}) ;{ }^{f}$ is the frequency of electromagnetic wave $(\mathrm{Hz}) ;{ }^{\sigma_{r}}$ is the relative conductivity $(\mathrm{S} / \mathrm{m}) ; \mu_{\mathrm{r}}$ is the relative permeability $(\mathrm{H} / \mathrm{m}) ; r$ is the distance from the source to the shield $(\mathrm{cm}) ; 5$ is skin depth $(\mathrm{cm})$.

The process of electromagnetic wave attenuation in the process of metal propagation, When the electromagnetic wave intensity attenuation to the surface of the original value of the penetrating $1 / e$ or $37 \%$ distance, namely the skin depth.

The skin depth is:

$$
\delta=\frac{2.6}{\sqrt{f \mu_{r} \sigma_{r}}}
$$

Skin depth is the theoretical basis for selecting shielding materials in the design process.

The skin depth of the common metals is shown in Table 2

Table 2 skin depth of common metals ( $\mathrm{mm})$

\begin{tabular}{l|lll}
\hline \multicolumn{1}{r}{ material } & brass & alumimium & ferrum \\
\cline { 2 - 3 } frequency & & & \\
\hline $50 \mathrm{~Hz}$ & 18.32 & 11.96 & 0.72 \\
$100 \mathrm{~Hz}$ & 12.95 & 8.46 & 0.51 \\
$1 \mathrm{KHz}$ & 4.10 & 2.67 & 0.16 \\
$10 \mathrm{KHz}$ & 1.30 & 0.85 & 0.051 \\
$150 \mathrm{KHz}$ & 0.33 & 0.22 & 0.013 \\
$1 \mathrm{MHz}$ & 0.13 & 0.085 & 0.0061 \\
$100 \mathrm{MHz}$ & 0.013 & 0.0085 & 0.0016 \\
$1.5 \mathrm{GHz}$ & 0.0033 & 0.0022 & 0.0013 \\
$10 \mathrm{GHz}$ & 0.0013 & 0.00085 & 0.0016 \\
\hline
\end{tabular}

From the above formula and analysis, it can be concluded that the shielding effectiveness of 
metal is as follows:

i. The higher frequency is, the greater absorption loss is; the higher metal permeability is, the greater absorption loss is ; the higher metal conductivity is, the greater the absorption loss; the thicker shield is ,the greater absorption loss is; increasing of the thickness of a skin depth, the absorption loss increased by about $9 \mathrm{~dB}$.

ii. When the thickness of the metal is $\delta$, absorption loss is $8.68 \mathrm{~dB}$, the intensity attenuation was $37 \%$; the metal thickness of the metal is $2.3 \delta$, absorption loss is $20 \mathrm{~dB}$, the intensity attenuation was $10 \%$; the metal thickness is $4.6 \delta$, absorption loss is $40 \mathrm{~dB}$, the intensity attenuation was $1 \%$.

iii. When the distance between the electromagnetic shielding and the shielding body is greater than $\frac{\lambda}{2 \pi}$, the electromagnetic wave is in the far field. For the far field, the higher the frequency of electromagnetic wave is, the less the reflection loss is. For the near field, the higher the electromagnetic wave frequency is, the smaller the reflection loss is.

\section{analysis of influence factors of electromagnetic shielding effectiveness of shelter}

In practical applications, the field source is not ideal for a short dipole or loop antenna, the impedance are not the same everywhere; in addition, aperture, ventilation and the door influence shielding effectiveness.

The design of electromagnetic shielding shelter, it relate to the quota of shelter strength, frequency, technology implementation process and cost consideration.

\section{Definiting thickness of the deck}

Shelter is usually used to shield structure of double-layer isolation skin with polyurethane foam in the middle distance, therefore, appropriate spacing of shelter isolation skin can inecrease shelding effectiveness.

When the interlamellar spacing is equal to the odd number of $1 / 4$ wavelength , $S E_{d B}=2\left(A_{d B 1}+R_{d B 1}\right)+6(d B) ;$ When the interlamellar spacing is equal to the evennumber of $1 / 4$ wavelength, $S E_{d B}=2 A_{d B 1}+R_{d B 1}(d B)$.Therefore, considering the stiffness and resonant factors, the board of internal and external skin spacing is appropriate for the $50 \mathrm{~mm}$.

\section{selection of shielding material and thickness}

The shielding material should have high conductivity and high permeability, but the actual material which has high conductivity and permeability does not exist. Copper and aluminum with good electrical conductivity, but the permeability is very low; and the permeability of iron nickel alloy is very high, but the conductivity is low. In practice, when the frequency is below $10 \mathrm{kHz}$, the skin depth of low carbon steel is much less than that of conductive material. the shielding material should bu based on the shielding effectiveness, selecting a reasonable thickness of the metal material, generally taking into account the difficulty of processing, shielding thickness should not be greater than $2 \mathrm{~mm}[3]$.

In practical application, sheilter make use of $1.2 \sim 1.5 \mathrm{~mm}$ aluminum currently; When the frequency is lower than $10 \mathrm{KHz}$, it is required to use $1.2 \sim 1.5 \mathrm{~mm}$ steel, aluminum shelter, and strictly 
to ensure the inner plate closed continuous topology.

\section{processing of shelter hole}

The shelter is not a closed shield, door, window and aperture, will destroy the shelter electrical continuity, the slit is not properly handled, the electromagnetic leakage is very serious. Therefore, these parts are the key factors to ensure the stability of shielding effectiveness

i. the electromagnetic leakage at the hole is caused by the wave impedance difference of the electromagnetic wave in the transmission path,The formula of shielding effectiveness is[4] :

$$
\mathrm{A}_{\mathrm{s}}=27.3 \frac{t}{l}+20 \lg \left(\frac{(1+N)^{2}}{4 N}\right)
$$

$A_{S}$ is the gap absorption loss, unit is $\mathrm{dB} ; t$ is the thickness of the material or the gap depth, the unit is $\mathrm{mm} ; l$ is the length of the gap, the unit is $\mathrm{mm} ; N$ is the ratio of the gap impedance and wave impedance. Therefore, the length, depth and the thickness of the material will affect the shielding effectiveness. The depth of the slot, the length of the gap and the thickness of the material are all the factors that affect the shielding effectiveness.

ii. in practice, in order to eliminate the discontinuity in the gap, it make use of welding or riveting. In order to further improve the shielding effectiveness, according to the contact surface characteristics and the frequency of electromagnetic wave, the gap can be filled shielding materials. Shielding material have Monel alloy[5], beryllium copper and tin copper wire shielding. When the frequency of electromagnetic wave is low than $1 \mathrm{GHz}$, it can use Monel alloy. reed material is generally use beryllium copper, it has good shielding effectiveness in low frequency shielding and high frequency shielding, but it easily damaged on excessive compression,

iii. when the diameter of the hole is larger than one wavelength, the hole can be regarded as a transmitting antenna. The shielding effectiveness is the worst when the aperture size is equal to the integral number of half wavelength, and the length of the gap or the diameter of the hole in the project is less than 1/100 1/10. The higher the frequency of the incident electromagnetic wave, the more attention should be paid to the problem.

iv. the surface flatness of gap caused by the overlap of the metal should be cleanliness , moreover to prevent the corrosion of different metals between the two. In practice, the use of alcohol to wipe the surface of the metal to remove oil and dust, and the metal surface plating treatment. Such as: steel and aluminum lap, in order to ensure the shielding effectiveness of low frequency, nickel plating can be treated.

\section{Conclusions}

In short, the shielding effectiveness is related to the selected material, the design method and the electromagnetic field environment in which the design method is the basis of the shielding effectiveness, and the process implementation is the guarantee of shielding effectiveness. In order to ensure the shielding effectiveness, the designer should choose the appropriate material according to the frequency of the incident electromagnetic wave and the shielding effectiveness index.To ensure the shielding effectiveness, the designer according to the frequency of the incident electromagnetic wave and shielding effectiveness indicators to select the appropriate material and reasonable layout, 
and select the appropriate composite materials. The process plan should ensure the indicators of design, such as: flatness, roughness, the level of surface treatment, etc.

\section{References}

[1]P. L. Rui, F.Wang:CommInforSyst and Tech Vol. 1, No.2(2011), p. 1 (In Chinese).

[2]X. K. Wang, Y. Qiu: Fire Control Radar Technology Vol.6, No. 4(2006), p. 35 (In Chinese)

[3]K.Duan: The Research of Electromagnetic Shielding Technology.(University of Electronic Science and Technology of China University, 2007) (In Chinese)

[4]T . He, Q. Li:New Tecnnology\&New Process .No.6(2013), p. 78 (In Chinese)

[5]C. G. Liu, T. H.Zhang:CommInforSyst and Tech Vol. 1, No.2(2010), p. 78 (In Chinese) 